\title{
Computer-Generated Holography for Dynamic Display of 3D Objects with Full Parallax
}

\author{
Xuewu Xu ${ }^{1}$, Sanjeev Solanki ${ }^{1}$, Xinan Liang ${ }^{1}$, Shuhong $\mathrm{Xu}^{2}$, Ridwan Bin Adrian Tanjung ${ }^{1}$, Yuechao Pan ${ }^{1}$, \\ Farzam Farbiz ${ }^{2}$, Baoxi $\mathrm{Xu}^{1}$ and Tow-Chong Chong ${ }^{1,3}$
}

\author{
${ }^{1}$ Data Storage Institute, $A *$ STAR (Agency for Science, Technology and Research), Singapore \\ ${ }^{2}$ Institute for Infocomm Research, A*STAR (Agency for Science, Technology and Research), Singapore \\ ${ }^{3}$ Department of Electrical and Computer Engineering, National University of Singapore
}

\begin{abstract}
In this paper a new holographic three-dimensional (3D) display system based on computer-generated hologram (CGH) is developed for the reconstruction of $3 \mathrm{D}$ objects with full parallax. A new algorithm is also developed to reduce the hologram computation time and memory usage. The dynamic 3D objects are successfully reconstructed at video rates in both real and virtual spaces.
\end{abstract}

Index Terms-Computer-Generated Hologram, Full Parallax, Holography, Three-Dimensional Display.

\section{INTRODUCTION}

Conventionally, in order to create three-dimensional (3D) effect, the most common method is to display rotating two-dimensional (2D) images on a 2D computer screen. This method only has psychological sense of depth but does not have physical depth. It does not have true space impression. This display technology is based on conventional computer graphics and image processing technique.

3D display is opening up a new era of future entertainment and will create high impact on our daily life. It will enable us to view 3D photos in virtual reality, play 3D games, watch 3D TV as if we're personally in that environment as screened, view and interact with 3D data in an intuitive manner. There are many 3D display technologies [1], which can usually be classified into four types: stereoscopic, auto-stereoscopic, volumetric and holographic

In stereoscopic displays, human binocular disparity is utilized to create 3D effects. Visual aid such as polarized or colored glasses is required to direct two different views to our left and right eyes, respectively. Our brain will combine these two different views to create the 3D effect. We also call it as aided-viewing stereoscopic technology. However, it cannot provide physical depth. Most 3D movies nowadays require users to wear special glasses that will limit our field of vision and may cause eye fatigue.

The principle of auto-stereoscopic technology is the same as that of stereoscopic one. However, it does not require any special glasses. It is known as free-viewing auto-stereoscopic technology. This technology is based on twin-view or multi-view LCD display with built-in projection techniques such as lenticular lenses and parallax barriers, which direct different images to specific viewing zone in space. Two eyes of the observer in front of the display screen will receive different images and create 3D effects. Usually, this display system only allows us to observe horizontal parallax of 3D objects through optics or head tracking by moving from left to right, and vice versa. It will be more complicated if vertical parallax is introduced. Another disadvantage of this technology is that the number of viewing zones is limited. It is not convenient for many people to observe $3 \mathrm{D}$ objects at the same time. Furthermore, due to fixed focus length, our eyes cannot change the focus length like that when viewing real objects, and it may also cause eye fatigue.

Volumetric display technology [2], [3] constructs 3D images in real 3D space by using many methods such as volumetric scanning, screen rotation and light projection techniques. One can observe this 3D image from almost any angle without eye fatigue. This display technology can be used in the fields such as medical imaging, mechanical computer-aided design and military visualization. However it cannot cause occlusion and essentially has fixed 3D volume.

Holographic display is a true 3D display technology [4], providing all four eye mechanisms (depth cues): binocular disparity, motion parallax, accommodation, and convergence. One can view 3D objects with full parallax displayed with holographic technology without wearing any special glasses and no visual fatigue will be caused to human eyes. The occlusion can be introduced and the reconstructed 3D images can be scaled to a desired size. Holographic 3D display products might be launched into the market with target applications like 3D photos, 3D games, and scientific visualization in the next 5-10 years. From long-term point of view, there is a potential to develop a new 3D TV system based on digital holographic display technology.

Recently, a joint project between DSI and I2R of A*STAR of Singapore has been initiated to develop a new holographic display system for the reconstruction of 3D objects. In this paper, we introduce our research progress in developing the holographic 3D display system with computer-generated holography (CGH). We also present a new algorithm that has been developed to reduce the hologram computation time and memory usage. The 3D objects have been dynamically displayed at video rates in both real and virtual spaces with our system currently developed. 


\section{RELATED WORK}

Holography can be used to record and reproduce the amplitude (luminance), wavelength (chroma) and phase differences of light waves via the interference and diffraction of coherent light. It has been successfully applied to the holographic video display systems [5], [6]. Holographic 3D display technology is based on computer-generated hologram (CGH) and spatial light modulator (SLM). A CGH is a digital hologram generated by computing the interference pattern between an imaginary object wave and a reference wave. The $\mathrm{CGH}$ is launched onto the SLM device. An expanded laser beam is then used to illuminate the SLM and to reconstruct the 3D image through light diffraction. Many people can view different perspectives of the reproduced 3D object at the same time in different angles without glasses. However, the practical application is hindered by the lack of algorithms that can generate high-resolution holograms fast enough. Therefore, it is still necessary to develop new algorithms that can greatly reduce the hologram computation time.

Various holographic 3D display systems have been developed by different R\&D groups in the past years [4], [7], [8]. Hilaire et al. [7] at MIT developed the holographic display system using diffraction-specific (DS) algorithm and multichannel acousto-optic modulator as an SLM. The computation time was much reduced when they implemented the DS algorithm on PC's graphics hardware. Maeno et al. [8] used liquid crystal devices (LCDs) as SLMs and discarded the vertical parallax. The performance of their system was limited by the LCD specifications such as pixel pitch and pixel number. Slinger et al. [4] at QinetiQ developed the first commercial level holographic display system using active tiling method in which they replicated EASLM (electronically addressed spatial light modulator) frames onto OASLM (optically addressed spatial light modulator). They implemented active tiling by introducing shutter system synchronized with EASLM frame rate to tile sub-holograms onto OASLM in correct sequence. The work done by Masuda et al. [9] at Chiba University was initially related to the development of new hardware for the fast computation of CGH using coherent ray tracing (CRT) method. Later they implemented CRT on PC's graphics hardware to improve CGH computation time. In our implementation, we used a digital micro-mirror device (DMD) as an SLM for 3D object reconstruction and performed CGH computation with a new algorithm.

\section{SYSTEM OVERVIEW AND DESCRIPTION}

We have developed a holographic 3D display system that allows us to view the reproduced 3D objects either through a 2D display screen in virtual space or via a 3D display medium in real space. A system block diagram is schematically shown in Fig. 1. A new algorithm has also been developed to reduce the computation time and memory usage for the CGHs of 3D objects. A detailed explanation of this algorithm will be given in section IV. Our 3D display system is based on ultra-high frame rate DMD (up to $8 \mathrm{kHz}$ ) for rendering holograms in real-time. The pixel size of DMD is $13.86 \mu \mathrm{m}$. Other off-the-shelf optical components and a $50 \mathrm{~mW}$ red laser diode

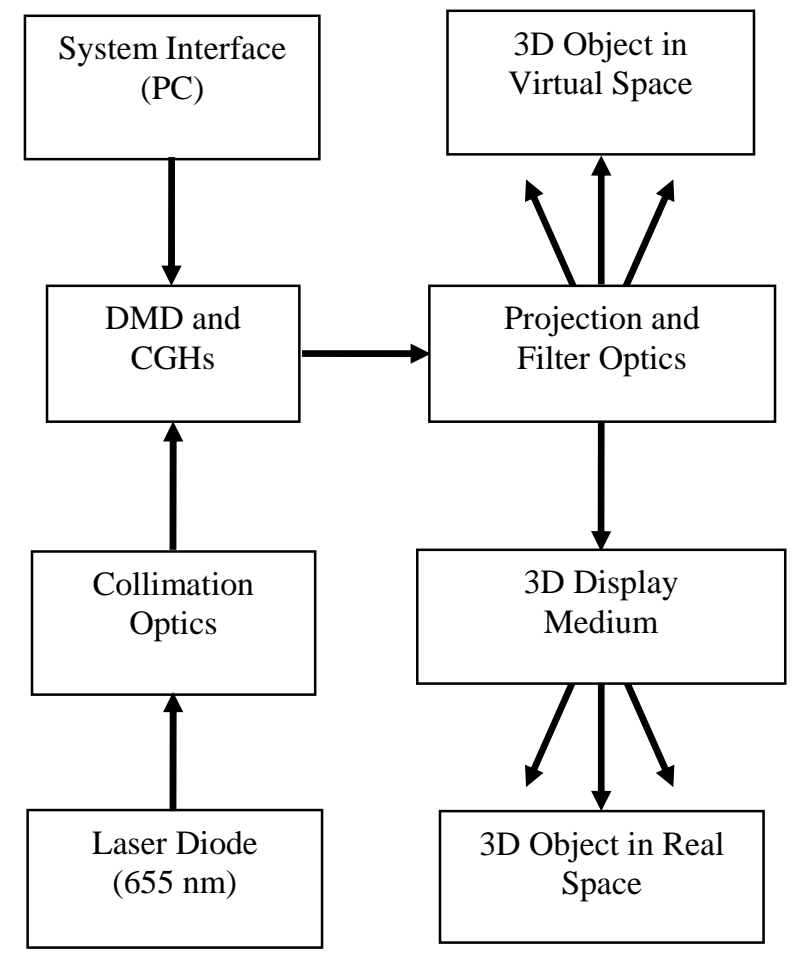

Fig. 1. Block diagram of holographic 3D display system.

at $655 \mathrm{~nm}$ are used to realize the reconstruction of 3D objects in virtual space as well as in real space. Two sets of optics are used. One is before DMD for cleaning, expanding and collimating laser beam as well as illuminating DMD with plane wave at required angle. The other is after DMD for guiding two types of reconstruction and filtering out the unwanted spatial frequency components in reconstructions. The reconstruction in virtual space is viewed by directly looking into the DMD. The reconstruction of 3D objects in real space is viewed by looking at $3 \mathrm{D}$ display medium such as gel tank or $2 \mathrm{D}$ paper on which the filtered 3D objects can be imaged at different depth locations in real space by free space propagation.

The system interface for control and display is developed by using LabView software. The simultaneous reconstruction of multiple 3D objects at different locations and the dynamic 3D object reconstruction at video rate are realized just by controlling the DMD frame rates with time-sequencing different 3D object holograms. This is enabled by using the DMD with an ultra-high frame rate up to $8 \mathrm{kHz}$ and a large on-board memory (16 GB). All other information regarding shape, size, and orientation of the 3D objects is pre-encoded into the holograms computed.

With our holographic 3D display system currently developed, we have reproduced dynamic 3D objects at video rates in both real and virtual spaces. Fig. 2 shows different perspectives of a $3 \mathrm{D}$ cuboid with the size of $1 \mathrm{~cm} \times 1 \mathrm{~cm} \times 2 \mathrm{~cm}$ reconstructed in real space, rotating along the vertical axis at different angles. 

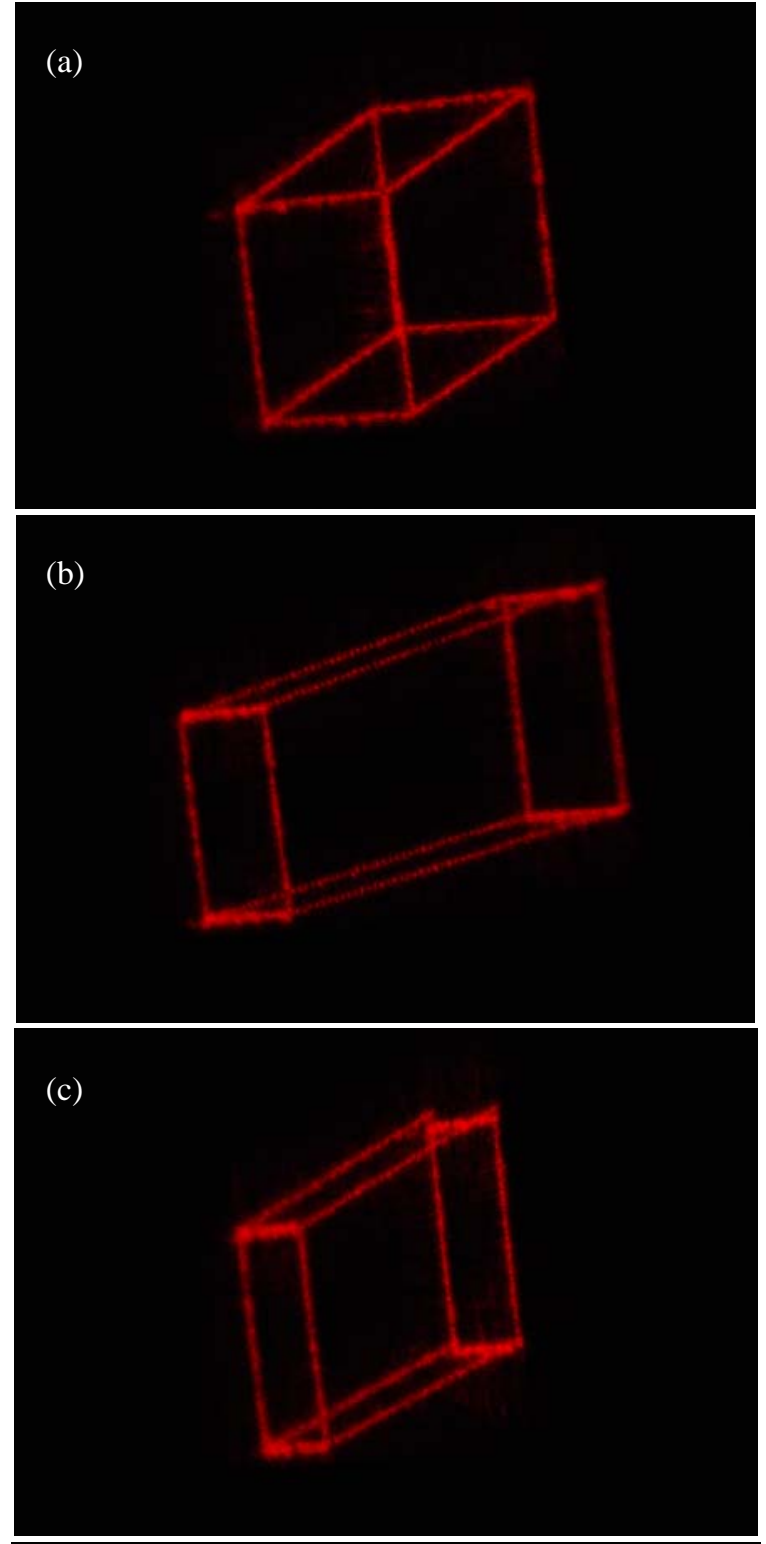

(d)

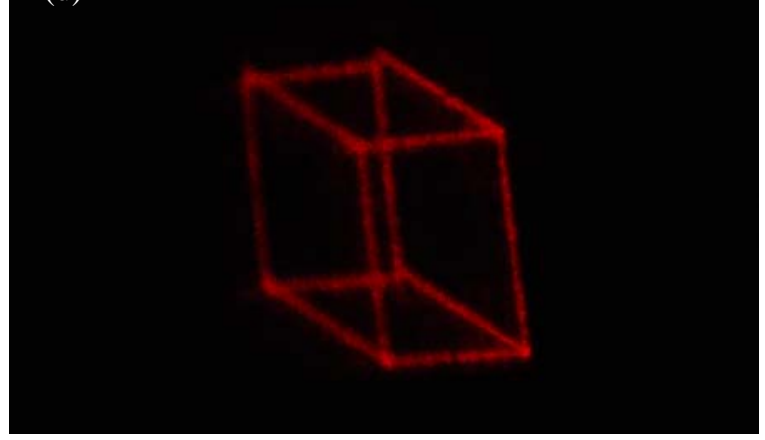

Fig. 2. Perspective of a rotating 3D cuboid reconstructed in real space with a rotating angle at (a) $0^{\circ}$, (b) $60^{\circ}$, (c) $120^{\circ}$ and (d) $180^{\circ}$.

\section{A NEW ALGORITHM FOR CGH COMPUTATION}

CGH computation is very time-consuming if the original point-to-point CRT approach is used [10]. The cuboid shown in Fig. 2 contains 520 sampling points in 3D space. It needs 64 seconds to compute CGH just for one single frame on an Intel QX9650 CPU. In order to reduce the computation time while maintaining the full parallax of 3D objects and low memory usage, we have developed a new algorithm and successfully implemented it in our CGH computation and holographic 3D display system.

Intensity distribution used in original CRT can be described as [9], [11]:

$$
I(x, y)=\sum_{j=1}^{N} a_{j} * \cos \left(\frac{2 \pi}{\lambda} \sqrt{\left(x-x_{j}\right)^{2}+\left(y-y_{j}\right)^{2}+z_{j}^{2}}\right),
$$

where $I(x, y)$ is the intensity in the hologram plane at $z=0, N$ the number of object points and $\lambda$ the wavelength. $\left(x_{j}, y_{j}, z_{j}\right)$ is the object point coordinates and $a_{j}$ the intensities.

One commonly used method to reduce computation time is to off-line pre-compute all possible values for the cosine function in (1), and store all results in a big table for further in-line computation. This algorithm is known as CRT with look-up table.

As in most of the cases, the object-hologram distance is much bigger than the wavelength (i.e. $z_{j}>>\lambda$ ), and also $z_{j}>>$ $\left(x-x_{j}\right),\left(y-y_{j}\right),(1)$ can be approximated as (2) using Fresnel approximations:

$I(x, y)=$

$\sum_{j=1}^{N} a_{j} * \cos \left(\frac{2 \pi}{\lambda} \sqrt{\left(x-x_{j}\right)^{2}+z_{j}^{2}}+\frac{2 \pi}{\lambda} \sqrt{\left(y-y_{j}\right)^{2}+z_{j}^{2}}\right)$

Rewrite (2) in complex form:

$I(x, y)=\sum{ }_{j=1}^{N} a_{j} * \operatorname{real}\left(e^{i(\theta+\varphi)}\right)$

$=\sum_{j=1}^{N} a_{j} * \operatorname{real}\left(e^{i \theta} * e^{i \varphi}\right)$

where $\theta=\frac{2 \pi}{\lambda} \sqrt{\left(x-x_{j}\right)^{2}+z_{j}^{2}}$ and $\varphi=\frac{2 \pi}{\lambda} \sqrt{\left(y-y_{j}\right)^{2}+z_{j}^{2}}$.

In (3), $\theta$ only depends on $x, x_{j}$ and $z_{j}$, while $\varphi$ only depends on $y, y_{j}$ and $z_{j}$. For a single object point, the resulted hologram is

$$
I_{j}(x, y)=a_{j} * \operatorname{real}\left(e^{i \theta} * e^{i \varphi}\right) \text {. }
$$

It is the product of three factors, object point intensity $a_{j}$, $\left(x_{j}, z_{j}\right)$ dependent factor $e^{i \theta}$ and $\left(y_{j}, z_{j}\right)$ dependent factor $e^{i \varphi}$. Physically, $e^{i \theta}$ modulates the beam in horizontal $(x)$ direction, and $e^{i \varphi}$ modulates the beam in vertical $(y)$ direction.

Thus, let horizontal light modulation factor

$$
H(x)=e^{i \theta}=e^{i\left(\frac{2 \pi}{\lambda} \sqrt{\left(x-x_{j}\right)^{2}+z_{j}^{2}}\right)},
$$

and vertical light modulation factor

$$
V(y)=e^{i \varphi}=e^{i\left(\frac{2 \pi}{\lambda} \sqrt{\left(y-y_{j}\right)^{2}+z_{j}^{2}}\right)},
$$

then (4) can be written as:

$$
I_{j}(x, y)=a_{j} * \operatorname{real}(H(x) * V(y)) \text {. }
$$

Examples of $H(x), V(y)$ and the resulted hologram $I_{j}(x, y)$ are shown in Fig. 3. 
(a)

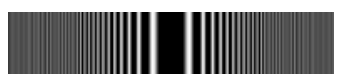

(c)

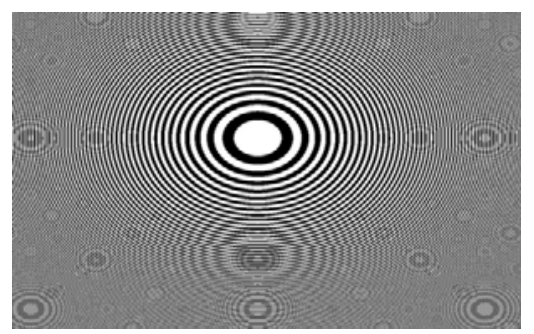

Fig. 3. Examples of (a) horizontal light modulation factor, (b) vertical light modulation factor and (c) resulted hologram.

Object points on the same vertical line share the same horizontal light modulation factor $H(x)$. According to (3) and (5), the hologram resulted from these object points can be written as:

$$
I^{\prime}(x, y)=\operatorname{real}\left(H(x) * \sum_{j=1}^{N} a_{j} * V(y)\right),
$$

where $n$ is the number of object points falling on the same vertical line, $a_{j}$ the intensities of these n object points, $I^{\prime}(x, y)$ the intensity in the hologram plane for these n object points.

The part $\sum_{j=1}^{n} a_{j} * V(y)$ in (6) is independent of $x$ and $x_{j}$, thus (6) can be broken down into two steps:

Step 1: $S(y)=\sum_{j=1}^{N} a_{j} * V(y)$,

Step 2: $I^{\prime}(x, y)=\operatorname{real}(H(x) * S(y))$.

where $S(y)$ is the sum of contribution in vertical direction from those $n$ object points to the hologram.

Assuming the width and height of hologram are $\mathrm{X}$ and $\mathrm{Y}$, computation complexity of (7) and (8) is in $\mathrm{O}(\mathrm{nY})$ and $\mathrm{O}(\mathrm{XY})$ respectively, where $\mathrm{O}(\mathrm{)})$ is the big $\mathrm{O}$ notation. Thus, for these $\mathrm{n}$ object points in the same vertical line, CGH computation complexity is in $\mathrm{O}(\mathrm{nY}+\mathrm{XY})$ using (7) and (8), while in $\mathrm{O}(\mathrm{nXY})$ using (1) or (3).

As number of possible $H(x)$ and $V(y)$ values are limited, they can be off-line pre-computed and stored in memory for in-line CGH computation. This can avoid using square and square root operations in in-line computation.

Detailed comparison of the number of operations for $n$ object points is listed in Table 1.

TABLE 1: COMPARISON OF THE NUMBER OF OPERATIONS AMONG ORIGINAL CRT, CRT WITH LOOK-UP TABLE AND THE NEW ALGORITHM.

\begin{tabular}{|c|c|c|c|}
\hline Operation & $\begin{array}{c}\text { Original } \\
\text { CRT }\end{array}$ & $\begin{array}{c}\text { CRT with } \\
\text { look-up table }\end{array}$ & $\begin{array}{c}\text { New } \\
\text { algorithm }\end{array}$ \\
\hline+- & $5 \mathrm{nXY}$ & $\mathrm{nXY}$ & $\mathrm{nY}+\mathrm{XY}$ \\
\hline$*$ & $5 \mathrm{nXY}$ & $\mathrm{nXY}$ & $\mathrm{nY}+\mathrm{XY}$ \\
\hline$\sqrt{ }$ & $\mathrm{nXY}$ & 0 & 0 \\
\hline $\cos$ & $\mathrm{nXY}$ & 0 & 0 \\
\hline
\end{tabular}

Both CRT with look-up table and the new algorithm show large reduction in computation load. When more than one object points fall on the same vertical line, the new algorithm has advantage over the one using look-up table.

The final hologram $I(x, y)$ in (3) for all $\mathrm{N}$ object points can be computed by summing up holograms $I^{\prime}(x, y)$ computed by (8) for all vertical lines which have object point(s) on them. Due to DMD properties, holograms have been binarized before they are launched onto DMD. The binarization is done in such a way that number of bright pixels and number of dark pixels are as close to equal as possible in resulted CGH.

Memory usages of these algorithms are listed in Table 2, assuming the whole object space is sampled into $m$ depth layers along $z$ axis.

TABLE 2: COMPARISON OF MEMORY REQUIREMENT AMONG ORIGINAL CRT, CRT WITH LOOK-UP TABLE AND THE NEW ALGORITHM

\begin{tabular}{|l|l|l|l|}
\hline Algorithm & $\begin{array}{l}\text { Original } \\
\text { CRT }\end{array}$ & $\begin{array}{l}\text { CRT with } \\
\text { look-up } \\
\text { table }\end{array}$ & $\begin{array}{l}\text { New } \\
\text { algorithm }\end{array}$ \\
\hline Memory & 0 & $\mathrm{mXY}$ & $\mathrm{m}(\mathrm{X}+\mathrm{Y})$ \\
\hline
\end{tabular}

Experimental comparisons on computation time and memory usage among algorithms are shown in Fig. 4 and Fig. 5 , respectively. The new algorithm increases computation speed by 30 times as compared with original CRT and around 3 times as compared to CRT with look-up table. It also reduces memory usage to $1 / 438$ as compared to CRT algorithm with look-up table for the hologram with the size of $1024 \times 768$. The new algorithm requires very low memory usage and hence almost overlaps with the line of the original CRT algorithm in Fig. 5. This set of data is based on a PC using Intel QX9650 CPU.

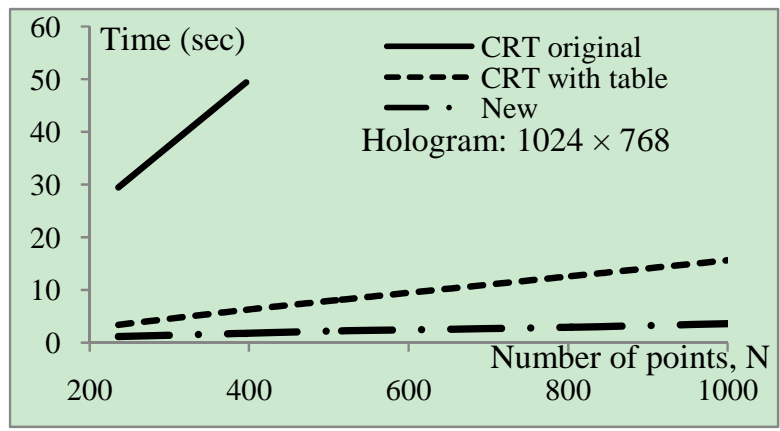

Fig. 4. Comparison of computation speed among algorithms.

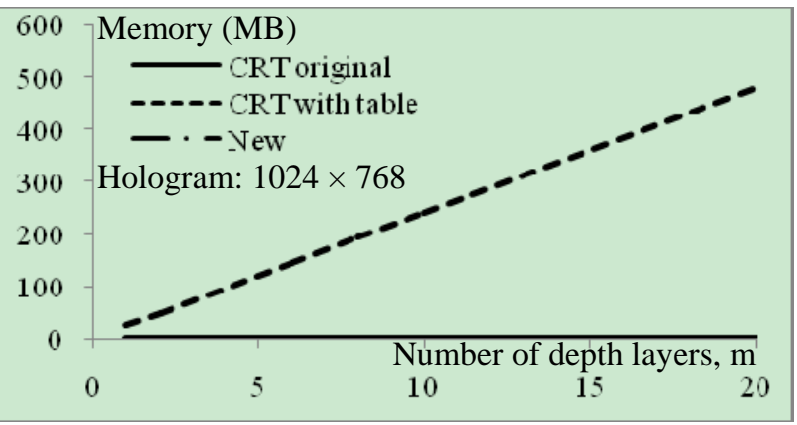

Fig. 5. Comparison of memory usage among algorithms. 


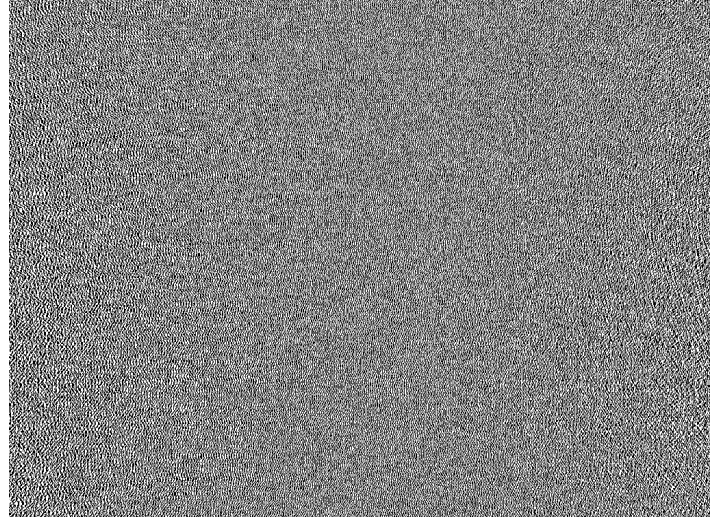

Fig. 6. Computer-generated hologram of a 3D cuboid.
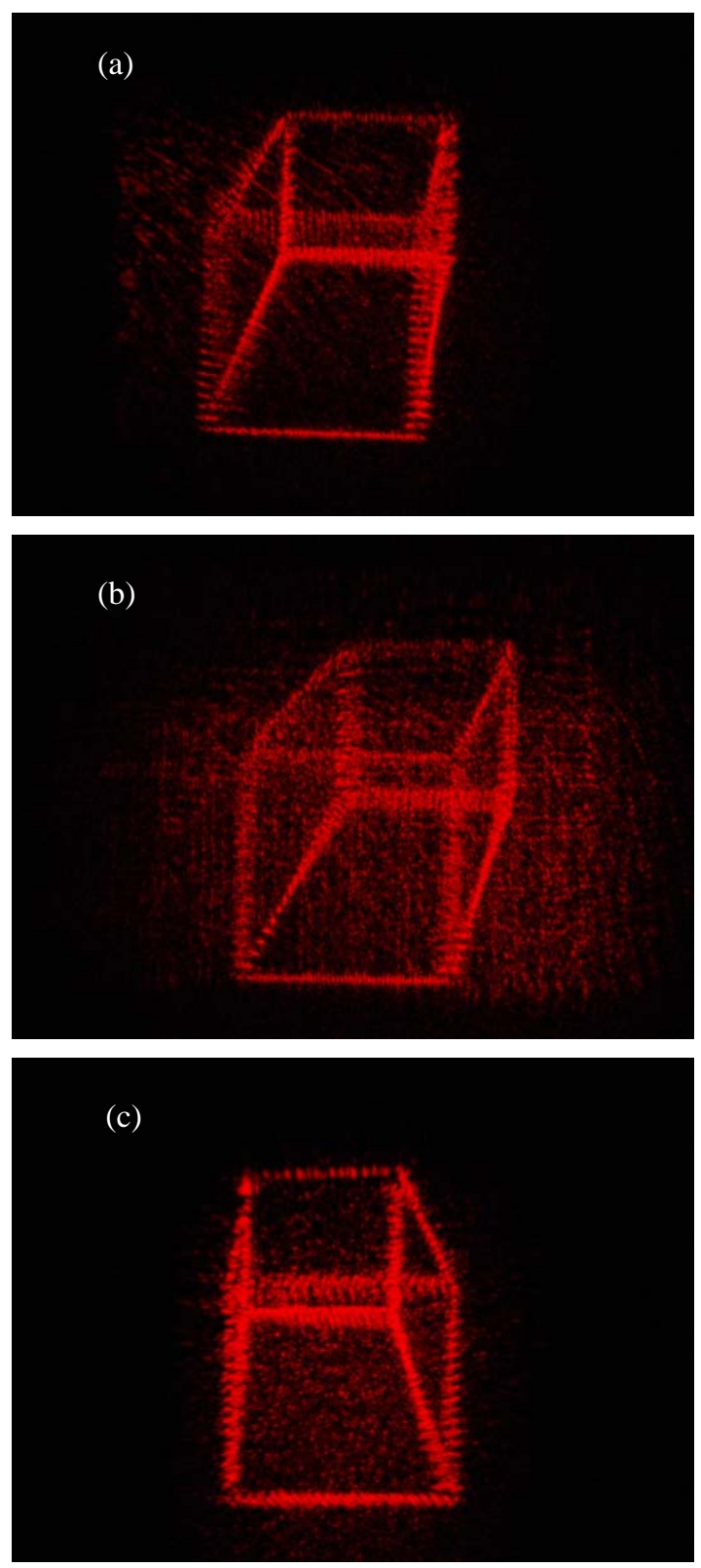

Fig. 7. 3D cuboid reconstructed in virtual space and viewed from (a) right, (b) center and (c) left, showing full parallax.
A typical binary CGH of a cuboid $(1 \mathrm{~cm} \times 1 \mathrm{~cm} \times 30 \mathrm{~cm})$ computed with the above new algorithm is shown in Fig. 6 . This CGH is 2D distribution of binary fringes decided by the object points in $3 \mathrm{D}$ space. As the CGH is to be displayed on DMD, its size is fixed to $1024 \times 768$, which is the same as DMD's resolution. Looking directly at this CGH does not provide any visual information unless it is illuminated with laser light with wavelength specified during computation.

Even though for virtual reconstruction the $3 \mathrm{D}$ object size is limited by SLM active window size ( 0.7 inch diagonal), for real reconstruction the object size is limited by projection distance only, i.e. real reconstructed object size is bigger than the object size used in CGH computation, when projected at far distance. The computed hologram has inherent lens function to create sharp 3D image at pre-decided distance.

The 3D object reconstructed from this CGH in virtual space with our holographic 3D display system is shown in Fig. 7(a), 7(b) and 7(c), which are corresponding to the perspectives viewed from right, center and left, respectively. We can see that the $3 \mathrm{D}$ cuboid has been reconstructed with full parallax.

Implementation and optimization of this new algorithm has also been done on graphic processing unit (GPU) and the computation speed has been increased by around 10 times as compared to that on CPU. Fig. 8 shows a typical 3D teapot reconstructed from its binarized holograms (CGHs) computed with our new algorithm on GPU. Dynamic display at video rate of such a 3D teapot rotating along its vertical axis has been realized with our holographic 3D display system.

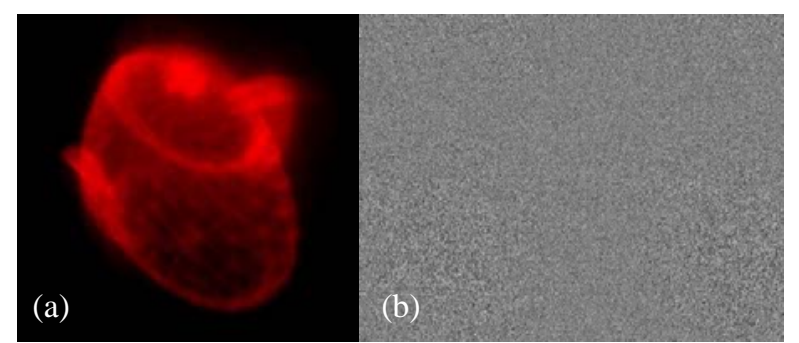

Fig. 8. (a) Typical 3D teapot reconstructed in virtual space and (b) its CGH.

Due to its less computational complexity and lower memory requirement, a few frames per second running speed on normal PC with GPUs and a few MBs of memory usage per CGH with thousands of object points are realized. It has shown the potential of this algorithm in producing full parallax and high quality 3D objects reconstructed at video rates. It may make holographic 3D display more suitable for handheld devices and home entertainment applications in the near future.

\section{CONCLUSION}

A holographic 3D display system is developed by using a DMD as an SLM, which allows us to view 3D objects either through a 2D display screen in virtual space or via a 3D display medium in real space. The dynamic display at video rate of 3D objects at different locations is realized with this system. A new algorithm is developed, which significantly reduces the time and memory usage for CGH computation. 


\section{ACKNOWLEDGEMENT}

We would like to thank the student, Ms $\mathrm{Ng}$ Li Ping from School of Physical and Mathematical Sciences of Nanyang Technological University of Singapore for her contribution to hologram computation. This project is funded by HOME2015 Programme of A*STAR, Singapore.

\section{REFERENCES}

[1] J. Y. Son, B. Javidi and K. D. Kwack. Methods for displaying three-dimensional images, in Proc. IEEE, vol. 94, pp. 502-523, 2006.

[2] D. Ebert, E. Bedwell, S. Maher, L. Smoliar and E. Downing. Realizing 3D visualization using crossed-beam volumetric displays, Communications of the ACM, vol. 42, no.8, pp. 101-107, Aug. 1999.

[3] G. E. Favalora. Volumetric 3D displays and application infrastructure, Computer, vol. 38, no. 8, pp. 37-44, Aug. 2005.

[4] C. Slinger, C. Cameron and M. Stanley. Computer-generated holography as a generic display technology, Computer, vol. 38, no. 8, pp. 46-53, 2005.

[5] T. Yamaguchi, G. Okabe and H. Yoshikawa. Real-time image plane full-color and full-parallax holographic video display system, Optical Engineering, vol. 46, pp. 125801-1 - 125801-8, 2007.

[6] Q. Y. J. Smithwick, D. E. Smalley, Jr. V. M. Bove and J. Barabas. Progress in holographic video displays based on guided-wave acousto-optic devices, in Proc. SPIE, vol. 6912, Practical Holography XXII: Materials and Applications, pp. 69120H-1 - 69120H-10, 2008.

[7] P. St. Hilaire, S. A. Benton, M. Lucente, J. Underkoffler and H. Yoshikawa. Real-time holographic display: improvements using a multichannel acoustio-optic modulator and holographic optical elements, in Proc. SPIE, vol. 1461, Practical Holography V, pp. 254-261, 1991.

[8] K. Maeno, N. Fukaya, O. Nishikawa, K. Sato and T. Honda. Electro-holographic display using 15 mega pixels LCD, in Proc. SPIE, vol. 2652, pp. 15-23, 1996.

[9] N. Masuda, T. Ito, T. Tanaka, A. Shiraki and T. Sugie. Computer generated holography using a graphics processing unit, Optics Express, vol. 14, no. 2, pp. 603-608, 2006

[10] C. D. Cameron, D. A. Pain, M. Stanley and C. W. Slinger. Computational challenges of emerging novel true 3D holographic displays, in Proc. SPIE, vol. 4109, pp. 129-140, July 2000.

[11] L. Ahremberg, P. Benzie, M. Magnor and J. Watson. Computer generated holography using parallel commodity graphics hardware, Optics Express, vol. 14, no. 17, pp. 7636-7641, 2006.

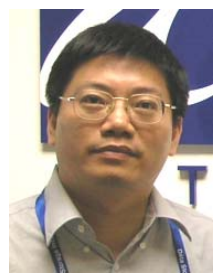

Xu Xuewu obtained his B.Sc. degree from Nanjing University and his Ph.D. degree from Chinese Academy of Sciences (CAS). He is a Research Scientist of Data Storage Institute. His research interests include holography for 3D display and high density data storage, holographic media and crystal materials. He is a member of The Society for Information Display and a member of International Organizing Committee of International Workshop on Holographic Memories \& Display.

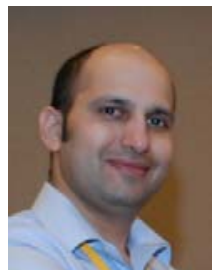

Sanjeev Solanki received his master degree from Indian Institute of technology, New Delhi, India and Ph.D in Electrical and Computer Engineering from National University of Singapore, Singapore. His research focus includes optical and electro - holography for application to high-density optical data storage and holographic TV.

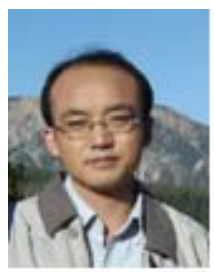

Liang Xinan is a Senior Research Fellow in Optica Materials \& System Division at Data Storage Institute (DSI), Agency for Science, Technology and Research (A*STAR) Singapore. He earned his M.Sc. in 1997 from Chinese Academy of Space Technology (CAST) and Ph.D. in 2000 from Chinese Academy of Science (CAS). His current research relates to holographic data storage media and holographic display technology.

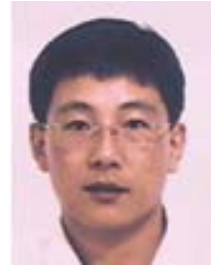

Xu Shuhong works as research scientist in the Institute for Infocomm Research, Singapore. He obtained his PhD in Mechanical Engineering from Zhejiang University (China). His research interests include: interactive 3D display, scientific visualization and computer-aided geometric design.

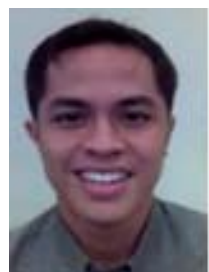

Ridwan Bin Adrian Tanjung (Singapore, 7/11/1981) graduated from National University Singapore in 2006 with a Bachelor of Engineering (2nd Class upper Honours) majoring in Electrical Engineering.

He was previously working for a semi-conductor company as a Process and Equipment Engineer before joining Data Storage Institute as a Research Engineer specializing in System Software Control.

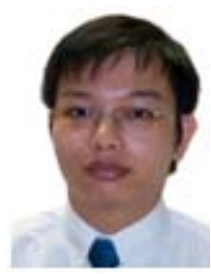

Pan Yuechao received his B.Eng. degree in computer engineering from National University of Singapore, 2008. He is currently a Research Engineer at Data Storage Institute, A*STAR, Singapore. His main research interest is in fast CGH computation and hardware integration for holographic 3D display system. He is a member of IEEE and ACM.

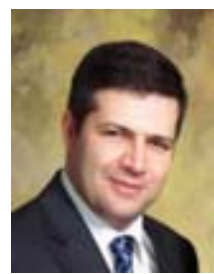

Farzam Farbiz (M’02) received his Ph.D. in 1999 from Amirkabir University of Technology, Tehran-Iran. His Ph.D. thesis was on computational intelligence filters for image enhancement. He received the first rank of the national young researcher award in 1999 as the best Iranian young researcher due to his research work.

From 2006 he has been working at A*STAR Institute for Infocomm Research as senior research fellow and principle investigator on multimodal game engine and mixed reality system for home application projects. He is also collaborating with A*STAR Data Storage Institute on developing laser holographic display systems. He has published more than 60 papers in international conferences and journals and has served as technical reviewer and program committee in many international journals and conferences.

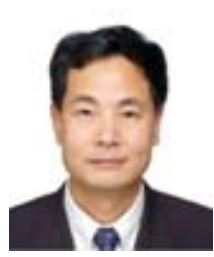

Xu Baoxi is Senior Scientist of Data Storage Institute. He received Ph.D. from Tsinghua University in electro-optics in 1994. He is with Data Storage Institute since 1995. His research interests include optical data storage, hybrid high density data storage, 3D display and surface Plasmon applications.

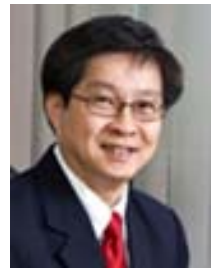

Chong Tow Chong obtained his B.Eng degree from the Tokyo Institute of Technology, his M.Eng degree from the National University of Singapore, and his Sc.D degree from the Massachusetts Institute of Technology, all in Electrical Engineering. He is currently the Executive Director of Science \& Engineering Research Council of A*STAR and Executive Director of the Data Storage Institute. Prof Chong's research interest is in the field of magnetic and optical data storage, especially in advanced thin films and devices for ultra-high density recording. His other research interests include high-speed electronic and optical devices. Prof Chong is also a Professor with the Department of Electrical and Computer Engineering, NUS. He has authored and co-authored more than 300 publications in international refereed journals, presented 23 invited talks and holds 20 patents. He serves as co-chairman of APMRC2008 and as member of the Technical Program Committee for ODS (USA), ISOM (Japan), APDSC, MORIS (Japan), CLEO Pacific (USA) and OECC (Japan). 